\title{
Inconsistent Reporting Between Meta-analysis Protocol and Publication - A Cross-Sectional Study
}

\author{
ALBERTO FALK DELGADO $^{1}$ and ANNA FALK DELGADO ${ }^{2}$ \\ ${ }^{1}$ Department of Surgical Sciences, Plastic and Reconstructive Surgery, Uppsala University, Uppsala, Sweden; \\ ${ }^{2}$ Department of Clinical Neuroscience, Karolinska Institute, Stockholm, Sweden
}

\begin{abstract}
Background: Inconsistent reporting in published meta-analyses compared to registered protocol are poorly understood. The aim of the study was to assess inconsistencies between registered protocols and published reports among oncology drug meta-analyses. Materials and Methods: A cross-sectional study was performed including oncology drug meta-analyses published between January 1st and November 14th 2016 with a published protocol. Two investigators extracted data on: selection criteria, outcome(s) and statistical plan in protocol and manuscript, plus self-acknowledgement of inconsistent reporting between protocol and publication. Results: Protocol registration was present in 19\% (23/119) of all oncology drug meta-analyses. In meta-analyses with protocol ( $n=23), 70 \%$ (16/23) had issues with inconsistent reporting between protocol and published report concerning; inclusion criteria, comparator group, intervention, outcome (PICO) or statistical analysis. Self-acknowledgement of changes between protocol and publication was found in 50\% (8/16). Conclusion: In metaanalyses with protocol, discrepancies between registered protocols and publications are frequent.
\end{abstract}

The medical community continuously strives to seek the latest and most beneficial treatment for its patients, while minimizing harm. In order to ensure the highest standards of care, the selection of treatment is preferably based on the aggregated scientific body of evidence available at that time point, rather than based on results from single trials (1). The usage of meta-analysis to summarize results from several studies has successively gained wide popularity. The increase

Correspondence to: Alberto Falk-Delgado, Uppsala University Hospital, Akademiska Sjukhuset, 75185 Uppsala, Sweden. Tel: +46 0186110000, Fax: +46 018133916, e-mail: alberto.falk-delgado@ surgsci.uu.se

Key Words: Meta-analysis protocol, inconsistent reporting, published report, trial registration. of meta-analyses ranges from one meta-analysis published in 1977 to 16,362 meta-analyses indexed in PubMed 2016 (2).

Selective and inconsistent reporting of randomized controlled trials (RCTs) are widely reported and recognized. At the RCT level, discrepancies between registered protocol and published manuscript with regards to outcome measures can pertain to the changing or omitting of primary endpoints, known as inconsistent or selective reporting (3-7). A potential consequence of inconsistent reporting is the publication of a seemingly positive trial powered for another (possibly not mentioned) outcome. Exploratory analyses of outcomes might cause false-positive results and problems with trial reproducibility, and mislead the readers of the importance of findings. In a clinical context, the worst-case scenario of the changing of an outcome might lead to drugs being approved for medical use based on a single study with a changed primary outcome $(8,9)$. Meta-analysis of high-quality RCTs is considered the highest level of evidence according to the Grades of Recommendation, Assessment, Development and Evaluation Working Group (GRADE) scale (10). However, the external validity of meta-analyses might be jeopardized due to selective and inconsistent reporting (11), with changes to the published manuscript other than those originally planned for (12). Furthermore, the retrospective nature of most meta-analyses might introduce bias with regards to insufficient blinding for study outcomes.

Several measures have been taken to increase transparency in the reporting of RCTs. Firstly, trial protocol registration in public databases is mandatory by the International Committee of Medical Journal Editors (ICMJE) since 2005 (13). A published protocol increases transparency for deviations from the protocol and makes it possible for anyone to assess. Despite the fact that meta-analysis protocol registration is now an available option (14), data on the usage of this option and the incidence of inconsistent reporting of meta-analyses is scarce. Furthermore, scientific journals of today encourage, but do not demand meta-analysis protocol registration (15).

In a Cochrane review aiming to study selective reporting at the systematic review level (review/meta-analysis protocol 
versus published review/meta-analysis), selective reporting of outcomes was found in $38 \%$ of the included systematic reviews (12). These results were further supported by the studies by Page et al. (1) and Tricco et al. (16). However, previous studies have been limited to systematic reviews, which might limit the generalizability to meta-analyses. Further, we are unaware of any previous study investigating the role of inconsistent reporting with regards to the full PICO (patient, intervention, comparison, outcome) outline and statistical analysis in meta-analyses (17).

Due to the lack of mandatory protocol registration for meta-analyses in most scientific journals we hypothesized that meta-analysis protocol registration might be low and inconsistencies between protocol and publication might be high. The aim of this study was to assess meta-analysis protocol registration and inconsistencies between study protocol and publication.

\section{Materials and Methods}

Reporting guidelines. This study was performed according to the Preferred Reporting Items for Systematic Reviews and MetaAnalyses (PRISMA) statement for reporting systematic reviews and meta-analyses of studies that evaluate health care interventions: explanation and elaboration (18). This study was not registered in Prospero since none of the outcomes were related to patient or clinical outcome (19).

\section{Criteria for inclusion in the study}

Eligibility criteria. We included published oncology drug metaanalyses with a previously published meta-analysis protocol. Oncology was defined as related to cancer treatment. Drug therapy was defined as systemic treatment related to cancer disease, including anti-cancer treatment, palliation or other drug therapy to improve symptoms related to cancer disease.

Data sources and search strategy. An electronic search was performed in PubMed. The search was restricted to PubMed in order to limit findings to established indexed journals. The search was conducted on Nov. 14th 2016 including controlled vocabulary for more precision. Searches were limited to dates between 2016/01/01 and 2016/11/14.

Inclusion and exclusion criteria. Oncology drug meta-analyses published in 2016 with a registered meta-analysis protocol were included. A meta-analysis protocol was defined as a protocol published in a publicly available database. Exclusion criteria at abstract and title level were: non-oncology meta-analysis, a systematic review without a meta-analysis, non-drug meta-analysis, non-meta-analysis, non-English text, review or commentary. Systematic reviews without meta-analysis were excluded since they do not require statistical analysis. Further, exclusion criteria at fulltext screening level were lack of protocol registration.

Study selection. The screening of studies was performed by one investigator (blinded for review), with reasons for exclusion discussed with a second investigator (blinded for review). Reference to a meta-analysis protocol was sought for in published manuscripts and supplementary material.
Data extraction. Data-extraction from protocol and published manuscript was performed independently by two investigators, in studies meeting all inclusion criteria. The following data was extracted: reported study selection criteria, primary outcome, secondary outcome(s) and statistical plan in protocol and manuscript, plus self-acknowledgement of inconsistent reporting between protocol and publication. Extracted study co-variables from publications were impact factor (20), funding source, conflict of interest, sex of the first author and origin of publication. First author sex determination was performed as previously reported (21).

Assessment of inconsistent reporting between meta-analysis protocol and publication. Assessment of inconsistent reporting was further evaluated through comparison between extracted data from protocol and published meta-analysis for each of the four domains based on PICO and statistical analysis. The first domain included "patient/population=type of study design", "intervention" and "comparator". The second domain included primary outcome, the third domain included secondary outcome and the fourth domain included statistical analysis. Inconsistent reporting was defined as any deviation in the published manuscript compared to the meta-analysis protocol. Inconsistent reporting in one domain was defined as "minor" inconsistency. Inconsistent reporting in two or more domains was defined as "major" inconsistency. Self-acknowledgement of changes was sought for in studies attributed with inconsistent reporting after evaluation of protocol and publication.

Quality assessment. Adherence to PRISMA guidelines was used as a surrogate marker for quality assessment (18). PRISMA covers 27 different domains, and adherence to all of these gave a maximum of 27 points. This score was used since the guidelines strive to improve reporting and quality of meta-analyses.

Main outcome variables. The main outcome variables were the presentation of a meta-analysis protocol in the published manuscript or supplementary material, and the frequency of inconsistent reporting in oncology drug meta-analyses.

Secondary outcome variables. Secondary outcome variables were: inconsistent reporting according to PICO and statistical analysis. Selfacknowledgement of inconsistent reporting in the published metaanalysis compared to the registered protocol was also evaluated.

Data synthesis and analysis. Summary statistics were used to describe the proportion of meta-analysis protocol registration, the characteristics of the included studies, and the prevalence and type of inconsistent reporting. Summary statistics were presented with $95 \%$ confidence intervals.

Role of the funding source. This study received no funding.

Patient involvement. No patients were involved in the study.

\section{Results}

Search results. The electronic search identified 283 hits that were screened for possible inclusion in this study. A total of 164 studies was excluded with reasons after full-text evaluation, and 119 oncology drug meta-analyses were identified as described by the flow-chart in Figure 1. Meta- 


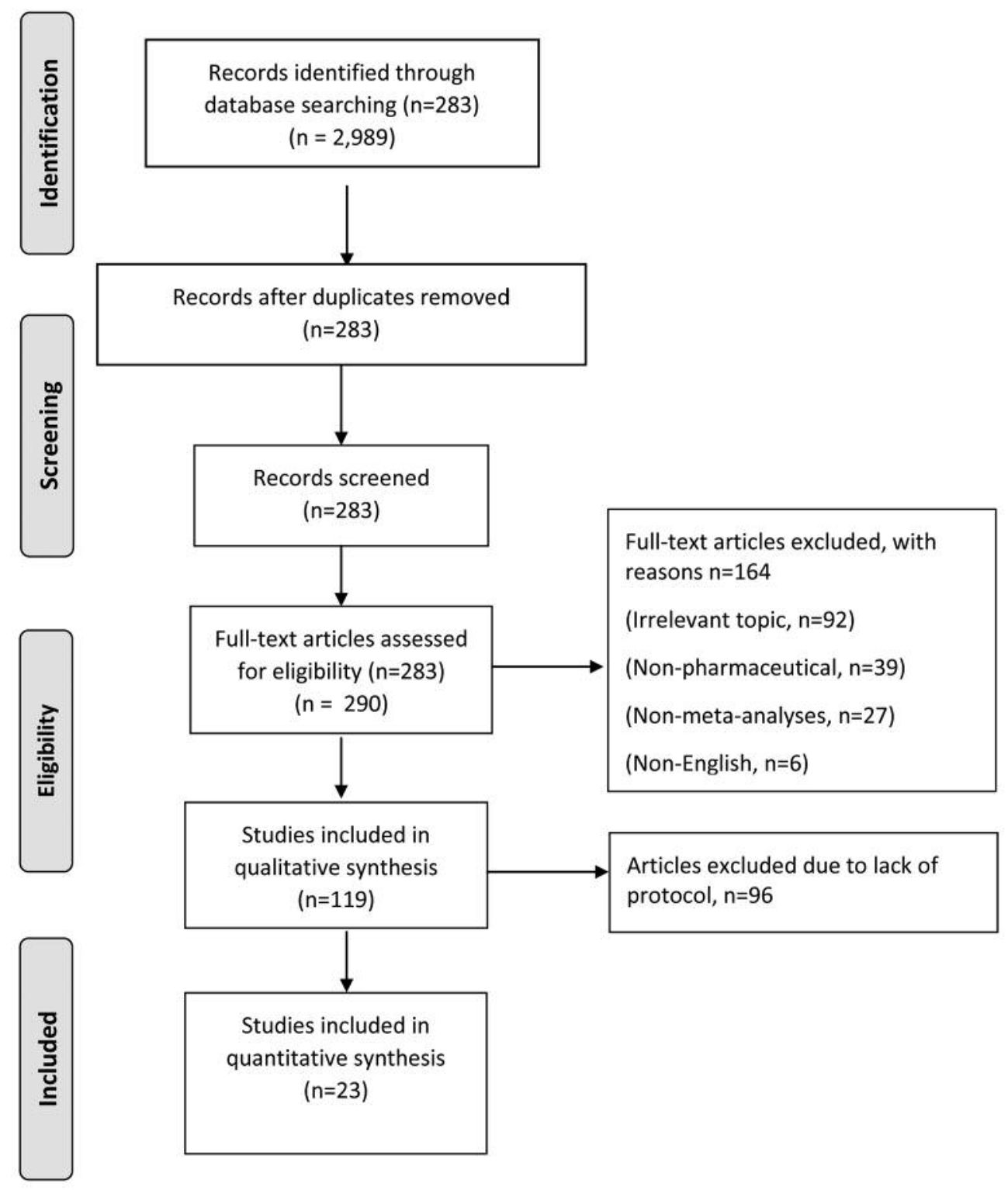

Figure 1. Trial search and selection according to PRISMA. PRISMA 2009 Flow Diagram (Moher D, Liberati A, Tetzlaff J, Altman DG, The PRISMA Group (2009). Preferred Reporting Items for Systematic Reviews and Meta-Analyses: The PRISMA Statement. PLoS Med 6(6): e1000097. doi:10.1371/journal.pmed1000097. For more information, visit www.prisma-statement.org).

analysis protocol registration was present in $23(19 \%, 95 \%$ CI, $13 \%$ to $27 \%$ ) studies (22-44). Excluding Cochrane reviews yielded a registration rate of $7 \%(\mathrm{CI}, 3 \%$ to $13 \%)$. Study characteristics and possible co-variables

A full description of included studies $(n=23)$ is presented in Table I. The majority of meta-analyses, 18 studies $(78 \%$, CI, $58 \%$ to $90 \%)$, were published in intermediate $(\geq 5, \leq 20)$ impact journals. Further, most of the meta-analyses, 16 (70\%, CI, $49 \%$ to $84 \%$ ), were Cochrane reviews. No meta- analysis was funded by a for-profit organization and a female first author was perceived in 11 (48\%, CI, 29\% to 67\%) studies. Risk of bias assessment revealed a mean adherence to PRISMA guidelines of 23 points.

Inconsistent reporting between meta-analysis protocol and publication. Inconsistent reporting between registered meta-analysis protocol and published meta-analysis was found in 16 (70\%, CI, $49 \%$ to $84 \%$ ) of 38 meta-analyses. 
Table I. Characteristics of the included meta-analyses with a published protocol.

\begin{tabular}{lc}
\hline Characteristics & $\mathrm{N}=23$ \\
\hline Impact factor (categorized) & No. $(\%)$ \\
High $(>20)$ & $1(4)$ \\
Intermediate $(\geq 5 \leq 20)$ & $18(78)$ \\
Low $(<5)$ & $4(18)$ \\
Conflict of interest & \\
Yes & $5(22)$ \\
No & $18(78)$ \\
Funding & \\
For-profit & 0 \\
Mixed-profit & 0 \\
Non-profit & $23(100)$ \\
Cochrane review & \\
Cochrane review & $16(70)$ \\
Non-cochrane & $7(30)$ \\
First author gender & \\
Female & $11(48)$ \\
Male & $12(52)$ \\
PAQS (maximum 27 points) & \\
$\leq 20$ & $3(13)$ \\
$>20$ & $20(87)$ \\
Origin of publication & \\
Asia & $9(39)$ \\
Australia & $2(9)$ \\
EU & $11(48)$ \\
USA & $1(4)$ \\
Study population & $\mathrm{IQR}$ \\
Median & $1719(645-2317)$ \\
\hline
\end{tabular}

PAQS, PRISMA adherence quality score; IQR, interquartile range.

Inconsistent reporting was then further categorized in four domains according to PICO and statistical analysis. Issues with domain one, including patient/population=study design (P), comparator (C) or intervention (I), were reported in $8(35 \%, \mathrm{CI}, 18 \%$ to $55 \%)$ studies and were most commonly related to the type of studies included in the meta-analysis $(\mathrm{P})$. Issues related to domain two, the primary outcome $(\mathrm{O} 1)$, were found in $5(22 \%, \mathrm{CI}, 10 \%$ to $42 \%$ ) of 23 meta-analyses and were mainly related to inadequate definition of the primary aim. Inconsistent reporting in domain three, which is the secondary aim $(\mathrm{O} 2)$, was present in 7 (30\%, CI, $16 \%$ to $51 \%)$ studies and most often related to the addition of a secondary aim in the published manuscript compared to the registered protocol. Issues related to domain four, the statistical plan, were found in $12(52 \%, \mathrm{CI}, 33 \%$ to $71 \%)$ meta-analyses. A summary of meta-analysis protocol registration and inconsistent reporting in the meta-analyses is reported in Table II.
Table II. Meta-analysis protocol and inconsistent reporting in four domains according to PICO and statistical analysis. Inconsistent reporting between meta-analysis protocol and publication.

\begin{tabular}{lc}
\hline & $\mathrm{N}(\%)$ \\
\hline Inconsistent reporting: any & \\
Yes & $16(70)$ \\
No & $7(30)$ \\
Inconsistent reporting: type & \\
$\quad$ Minor* & $9(39)$ \\
Major** & $7(31)$ \\
Inconsistent reporting of inclusion criteria: & \\
Patient, Intervention, Comparison (PICO) & \\
Yes & $7(30)$ \\
No & $16(70)$ \\
Inconsistent reporting of primary outcome (PICO1) & \\
$\quad$ Yes & $5(22)$ \\
No & $18(78)$ \\
Inconsistent reporting of secondary outcome (PICO2) & \\
Yes & $7(30)$ \\
No & $16(70)$ \\
Inconsistent reporting of statistical analysis & $12(52)$ \\
Yes & $11(48)$ \\
Self-acknowledgement of inconsistent & \\
reporting in manuscript in studies with & \\
inconsistent reporting (n=16) & \\
Yes & \\
No & $8(50)$ \\
\hline
\end{tabular}

Domain one denotes $\mathrm{P}=$ patient, $\mathrm{I}=$ intervention, $\mathrm{C}=$ comparison. Domain two denotes the primary outcome (O1). Domain three denotes the secondary outcome $(\mathrm{O} 2)$. Domain four denotes the statistical plan. *Inconsistent reporting in one domain, **inconsistent reporting in at least two domains.

\section{Discussion}

Prospective registration of meta-analysis protocol might increase awareness of the scientific value of pre-specified analyses and study conclusions based on this. As of today, registration of meta-analysis protocol is not mandatory, but might be encouraged in certain journals (15). Our study shows that there is a low prevalence of prospective metaanalysis protocols among oncology drug meta-analyses. We found that significant alterations are common when comparing the registered protocol with the published manuscript. Inconsistent reporting related to the statistical analysis occurred in more than half of included studies. Furthermore, self-acknowledgement of changes was found in only $50 \%(8 / 16)$ of meta-analyses attributed with inconsistent reporting.

To the best of our knowledge, this is the first study to assess several aspects other than inconsistent reporting of the primary outcome between meta-analysis protocol and published meta- 
analysis (12). Based on the financial incentives associated with cancer drugs, we decided a priori to limit our study to oncology drug meta-analyses. The cost for these drugs is rapidly increasing $(45,46)$, though this increase is not related to more clinically-efficient drugs (47). Elevation of oncology drug price has been widely discussed in the medical community (19). We did not restrict our study only to anticancer treatment, but included all pharmacological systemic treatments related to cancer patient therapies. Furthermore, we decided to focus on meta-analyses since they combine statistical results as opposed to systematic reviews. Combining results might potentially lead to bias when adding, omitting or changing subgroups or sensitivity analyses. However, this study has certain limitations. Importantly, most non-Cochrane meta-analyses lack protocol, thus the frequency of inconsistent reporting in these might be underestimated. Limiting this cross-sectional study to oncology might lower generalizability to other therapeutic areas.

Systematic review registration was reported to $16 \%$ in February 2014 (1). In this study we report a prevalence of meta-analysis protocol registration of $19 \%$. While these data suggest that prospective protocol registration is still at a low level, the true evolution of meta-analysis protocol registration is unknown due to lack of previously published similar studies with comparable data. The prevalence of inconsistent reporting of primary outcomes among systematic reviews has been reported between 22-47\%. However, several studies have been limited to Cochrane reviews published between 2000-2009, before the publication of PRISMA guidelines (4850 ), which is supported by a more recent publication restricted to systematic reviews with a Prospero protocol (16). In our study, we found inconsistent reporting of primary outcomes in $22 \%$ of included studies. Concerning inconsistent reporting of secondary outcomes, their prevalence is assessed at $17 \%$ of Cochrane reviews (49). We reported changes of secondary outcomes in $30 \%$ of the metaanalyses. In a recent study it was found that adverse outcomes were missing or partially reported in $86 \%$ of the reviews from the included randomized and non-randomized trials (51). PRISMA guidelines recommended that changes to systematic review after the review started should be reported. Further, Cochrane introduced a subheading entitled "Differences between protocol and review" in 2008, in order to clarify any deviations from the protocol (18). However, selfacknowledged changes to the published manuscript compared to the protocol was low.

A previous study has shown low reproducibility rate (11\%) of the findings of "landmark" oncology studies (52). This has been further supported by a replication study assessing published oncology and cardiovascular findings (53). Problems with inadequate usage of statistical tests might also contribute to the low replication rates (54). Prospectively published meta-analysis protocols allow the public to compare it with the published meta-analysis and make its own decision on deviations from the protocol. It has previously been suggested that meta-analysis protocol registration could improve the reporting of meta-analysis (55).

Our study shows that the rate of prospective meta-analysis protocols is unsatisfactorily low. Registered protocols were found in only $7 \%$ of the meta-analyses when we excluded Cochrane reviews. Given the rapid increase of the use of meta-analyses in scientific evidence synthesis, we believe that active measures have to been taken in order to reduce reporting bias. We suggest that meta-analysis protocol registration should be demanded by the ICMJE as previously has been implemented with RCTs through the introduction of the CONSORT guidelines $(56,57)$.

We used the PRISMA guidelines adherence as a surrogate marker for quality (18), since PRISMA charts are often required at the stage of submission to many journals. PRISMA adherence should ideally be $100 \%$ in metaanalysis. We found a mean adherence to PRISMA of $89 \%$. This fact could easily be improved by an automatic software screening tool, which could help authors and editors to improve reporting according to PRISMA guidelines $(58,59)$.

Bias related to issues with inconsistent reporting might occur at several levels. Bias might be introduced with patient inclusion, comparator, intervention, outcome or statistical analysis. Avoiding selective reporting at these levels might lead authors to less biased summary estimates, thus reducing misinterpretation of the available scientific evidence. We do acknowledge that a pre-specified outcome or subgroup analysis might not be possible to perform, due to the lack of data in included studies, and therefore not acknowledging this as inconsistent reporting in our study. Also, meta-analyses are retrospective by nature, and therefore an identical overlapping meta-analyses protocol with a published meta-analysis might imply that the study protocol in fact was performed after the study was conducted. In meta-analyses with poorly specified outcomes in the protocol, a more precise definition of the outcome could increase the transparency in order for the reader to assess the presence of inconsistent reporting. Clear definitions of study outcomes could be demanded by public meta-analysis registries, such as PROSPERO, before being made publicly available. Further, we suggest that the PRISMA guidelines should be updated with the addition of a subheading concerning self-acknowledgement of changes made from protocol.

Our results support that it is not sufficient only to encourage the registration of meta-analysis protocol before starting the meta-analysis. On the contrary, this work highlights the need for medical editors and scientific journals to demand meta-analysis protocol registration during the editorial process when reviewing the growing number of meta-analyses, in order to detect not acknowledged or unjustified changes between protocol and publication. 


\section{Conclusion}

Meta-analysis protocol registration among oncology drug meta-analyses is uncommon. We found a high rate of discordance between meta-analyses protocol registries and published meta-analyses.

\section{References}

1 Page MJ, Shamseer L, Altman DG, Tetzlaff J, Sampson M, Tricco AC, Catalá-López F, Li L, Reid EK, Sarkis-Onofre R and Moher D: Epidemiology and reporting characteristics of systematic reviews of biomedical research: A cross-sectional study. PLoS Med 13: e1002028, 2016.

2 US National Library of Medicine National Institutes of Health. http://www.pubmed.com/ Accessed 2017 Feb 28.

3 Dwan K, Altman DG, Cresswell L, Blundell M, Gamble CL and Williamson PR: Comparison of protocols and registry entries to published reports for randomised controlled trials. Cochrane Database Syst Rev 1: MR000031, 2011.

4 You B, Gan HK, Pond G and Chen EX: Consistency in the analysis and reporting of primary end points in oncology randomized controlled trials from registration to publication: a systematic review. J Clin Oncol 30: 210-216, 2012.

5 Mathieu S, Boutron I, Moher D, Altman DG and Ravaud P: Comparison of registered and published primary outcomes in randomized controlled trials. JAMA 302(9): 977-984, 2009.

6 Becker JE, Krumholz HM, Ben-Josef G and Ross JS: Reporting of results in ClinicalTrials gov and high-impact journals. JAMA. 311: 1063-1065, 2014.

7 Hartung DM, Zarin DA, Guise JM, McDonagh M, Paynter R and Helfand $\mathrm{M}$ : Reporting discrepancies between the ClinicalTrials.gov results database and peer-reviewed publications. Ann Intern Med 160: 477-483, 2014.

8 Sridhara R, Johnson JR, Justice R, Keegan P, Chakravarty A and Pazdur R: Review of oncology and hematology drug product approvals at the US Food and Drug Administration between July 2005 and December 2007. J Natl Cancer Inst 102: 230-243, 2010.

9 Schwartz LM, Woloshin S, Zheng E, Tse T and Zarin DA: ClinicalTrials.gov and Drugs@FDA: A comparison of results reporting for new drug approval trials. Ann Intern Med 165: 421430, 2016.

10 Guyatt GH, Oxman AD, Vist GE, Kunz R, Falck-Ytter Y, Alonso-Coello P, Schünemann HJ and GRADE Working Group: GRADE: an emerging consensus on rating quality of evidence and strength of recommendations. BMJ 336: 924-926, 2008.

11 Williamson PR, Gamble C, Altman DG and Hutton JL: Outcome selection bias in meta-analysis. Stat Methods Med Res 14: 515524, 2005.

12 Page MJ, McKenzie JE, Kirkham J, Dwan K, Kramer S, Green $\mathrm{S}$ and Forbes A: Bias due to selective inclusion and reporting of outcomes and analyses in systematic reviews of randomised trials of healthcare interventions. Cochrane Database Syst Rev 10: MR000035, 2014.

13 De Angelis C, Drazen JM, Frizelle FA, Haug C, Hoey J, Horton R, Kotzin S, Laine C, Marusic A, Overbeke J, Schroeder TV, Sox HC and Van Der Weyden MB: Clinical trial registration: a statement from the International Committee of Medical Journal Editors. N Engl J Med 351: 1250-1251, 2004.
14 Booth A, Clarke M, Ghersi D, Moher D, Petticrew M and Stewart L: Establishing a minimum dataset for prospective registration of systematic reviews: an international consultation. PLoS One 6: e27319, 2011.

15 Annals of Internal Medicine. www.annals.org. Accessed 24 March, 2017.

16 Tricco AC, Cogo E, Page MJ, Polisena J, Booth A, Dwan K, MacDonald H, Clifford TJ, Stewart LA, Straus SE and Moher D: A third of systematic reviews changed or did not specify the primary outcome: a PROSPERO register study. J Clin Epidemiol 79: 46-54, 2016.

17 Higgins JPT G Se. Cochrane Handbook for Systematic Reviews Interventions. The Cochrane Collaboration.Version 5.1.0 (updated March 2011), 2011.

18 Liberati A, Altman DG, Tetzlaff J, Mulrow C, Gøtzsche PC, Ioannidis JP, Clarke M, Devereaux PJ, Kleijnen J and Moher D: The PRISMA statement for reporting systematic reviews and meta-analyses of studies that evaluate health care interventions: explanation and elaboration. Ann Intern Med 151(4): W65-94, 2009.

19 Prospero International Prospective Register of Systematic Reviews. https://www.crd.york.ac.uk/PROSPERO/.

$20 \mathrm{https}: / /$ jcr.incites.thomsonreuters.com/

21 Jagsi R, Guancial EA, Worobey CC, Henault LE, Chang Y, Starr $\mathrm{R}$, Tarbell NJ and Hylek EM: The "gender gap" in authorship of academic medical literature - a 35 -year perspective. N Engl J Med 355(3): 281-287, 2006.

22 Chen L, Chen C, Yen Y and Tam KW: Chemotherapy for advanced biliary tract carcinoma: A meta-analysis of randomized controlled trials. Medicine (Baltimore) 95(33): e4584, 2016.

23 Jin X, Ruiz Beguerie J, Sze DM and Chan GC: Ganoderma lucidum (Reishi mushroom) for cancer treatment. Cochrane Database Syst Rev 4: CD007731, 2016.

24 Wuntakal R, Seshadri S, Montes A and Lane G: Luteinising hormone releasing hormone (LHRH) agonists for the treatment of relapsed epithelial ovarian cancer. Cochrane Database Syst Rev 6: CD011322, 2016.

25 Lawrie TA, Alazzam M, Tidy J, Hancock BW and Osborne R: First-line chemotherapy in low-risk gestational trophoblastic neoplasia. Cochrane Database Syst Rev 6: CD007102, 2016.

26 Greenhalgh J, Dwan K, Boland A, Bates V, Vecchio F, Dundar Y, Jain P and Green JA: First-line treatment of advanced epidermal growth factor receptor (EGFR) mutation positive nonsquamous non-small cell lung cancer. Cochrane Database Syst Rev 5: CD010383, 2016.

27 Moriwaki T, Yamamoto Y, Gosho M, Kobayashi M, Sugaya A, Yamada T, Endo S and Hyodo I: Correlations of survival with progression-free survival, response rate, and disease control rate in advanced biliary tract cancer: a meta-analysis of randomised trials of first-line chemotherapy. Br J Cancer 114(8): 881-888, 2016.

28 Best LM, Mughal M and Gurusamy KS: Non-surgical versus surgical treatment for oesophageal cancer. Cochrane Database Syst Rev 3: CD011498, 2016.

29 van Dalen EC, van der Pal HJ and Kremer LC: Different dosage schedules for reducing cardiotoxicity in people with cancer receiving anthracycline chemotherapy. Cochrane Database Syst Rev 3: CD005008, 2016.

30 Chung VC, Wu X, Lu P, Hui EP, Zhang Y, Zhang AL, Lau AY, Zhao J, Fan M, Ziea ET, Ng BF, Wong SY and Wu JC: Chinese Herbal medicine for symptom management in cancer palliative 
care: systematic review and meta-analysis. Medicine (Baltimore) 95(7): e2793, 2016.

31 Hutzschenreuter F, Monsef I, Kreuzer KA, Engert A and Skoetz $\mathrm{N}$ : Granulocyte and granulocyte-macrophage colony stimulating factors for newly diagnosed patients with myelodysplastic syndromes. Cochrane Database Syst Rev 2: CD009310, 2016.

32 Wulaningsih W, Wardhana A, Watkins J, Yoshuantari N, Repana D and Van Hemelrijck M: Irinotecan chemotherapy combined with fluoropyrimidines versus irinotecan alone for overall survival and progression-free survival in patients with advanced and/or metastatic colorectal cancer. Cochrane Database Syst Rev 2: CD008593, 2016.

33 Lamarca A, Elliott E, Barriuso J, Backen A, McNamara MG, Hubner R and Valle JW: Chemotherapy for advanced nonpancreatic well-differentiated neuroendocrine tumours of the gastrointestinal tract, a systematic review and meta-analysis: A lost cause? Cancer Treat Rev 44: 26-41, 2016.

34 Mhaskar R, Wao H, Miladinovic B, Kumar A and Djulbegovic $\mathrm{B}$ : The role of iron in the management of chemotherapy-induced anemia in cancer patients receiving erythropoiesis-stimulating agents. Cochrane Database Syst Rev 2: CD009624, 2016.

35 Phillips RS, Friend AJ, Gibson F, Houghton E, Gopaul S, Craig JV and Pizer B: Antiemetic medication for prevention and treatment of chemotherapy-induced nausea and vomiting in childhood. Cochrane Database Syst Rev 2: CD007786, 2016.

36 Jaaback K, Johnson $\mathrm{N}$ and Lawrie TA: Intraperitoneal chemotherapy for the initial management of primary epithelial ovarian cancer. Cochrane Database Syst Rev 1: CD005340, 2016.

37 Tangjitgamol S, Manusirivithaya S, Laopaiboon M, Lumbiganon $\mathrm{P}$ and Bryant A: Interval debulking surgery for advanced epithelial ovarian cancer. Cochrane Database Syst Rev 1: CD006014, 2016.

38 Vale CL, Burdett S, Rydzewska LH, Albiges L, Clarke N, Fisher D2, Fizazi K, Gravis G, James ND, Mason MD, Parmar MK, Sweeney CJ, Sydes MR, Tombal B and Tierney JF: Addition of docetaxel or bisphosphonates to standard of care in men with localised or metastatic, hormone-sensitive prostate cancer: a systematic review and meta-analyses of aggregate data. Lancet Oncol 17(2): 243-256, 2016.

39 Bao YJ, Hou W, Kong XY, Yang L, Xia J, Hua BJ and Knaggs R: Hydromorphone for cancer pain. Cochrane Database Syst Rev 10: CD011108, 2016.

40 Scott K, Hayden PJ, Will A, Wheatley K and Coyne I: Bortezomib for the treatment of multiple myeloma. Cochrane Database Syst Rev 4: CD010816, 2016.

$41 \mathrm{Ma}$ H, Tian X, Zeng XT, Zhang Y, Wang Y, Wang F and Zhou JG: The efficacy of erlotinib versus conventional chemotherapy for advanced nonsmall-cell lung cancer: A PRISMA-compliant systematic review with meta-regression and meta-analysis. Medicine (Baltimore) 95(2): e2495, 2016.

42 Ma H, Liu Y, Huang L, Zeng XT, Jin SH, Yue GJ, Tian X and Zhou JG: The adverse events of oxycodone in cancer-related pain: a systematic review and meta-analysis of randomized controlled trials. Medicine (Baltimore) 95(15): e3341, 2016.

43 Chen X, Deng L, Jiang X and Wu T: Chinese herbal medicine for oesophageal cancer. Cochrane Database Syst Rev 1: CD004520, 2016.

44 Song H, Zhu J and Lu D: Molecular-targeted first-line therapy for advanced gastric cancer. Cochrane Database Syst Rev 7: CD011461, 2016.
45 Kantarjian HM, Fojo T, Mathisen M and Zwelling LA: Cancer drugs in the United States: Justum Pretium - the just price. J Clin Oncol 31(28): 3600-3604, 2016.

46 Experts in Chronic Myeloid L: The price of drugs for chronic myeloid leukemia (CML) is a reflection of the unsustainable prices of cancer drugs: from the perspective of a large group of CML experts. Blood 121(22): 4439-4442, 2013.

47 Mailankody S and Prasad V: Five years of cancer drug approvals: innovation, efficacy, and costs. JAMA Oncol 1(4): 539-540, 2016.

48 Silagy CA, Middleton P and Hopewell S: Publishing protocols of systematic reviews: comparing what was done to what was planned. JAMA 287(21): 2831-2834, 2002.

49 Kirkham JJ, Altman DG and Williamson PR: Bias due to changes in specified outcomes during the systematic review process. PLoS One 5(3): e9810, 2010.

50 Dwan K, Kirkham JJ, Williamson PR and Gamble C: Selective reporting of outcomes in randomised controlled trials in systematic reviews of cystic fibrosis. BMJ Open 3: e002709, 2013.

51 Saini P, Loke YK, Gamble C, Altman DG, Williamson PR and Kirkham JJ: Selective reporting bias of harm outcomes within studies: findings from a cohort of systematic reviews. BMJ 349 : g6501, 2014.

52 Begley CG and Ellis LM: Drug development: Raise standards for preclinical cancer research. Nature 483(7391): 531-533, 2014.

53 Prinz F, Schlange T and Asadullah K: Believe it or not: how much can we rely on published data on potential drug targets? Nat Rev Drug Discov 10(9): 712, 2011.

54 Garcia-Berthou E and Alcaraz C: Incongruence between test statistics and $p$-values in medical papers. BMC Med Res Methodol 4: 13, 2004.

55 Ioannidis JP, Greenland S, Hlatky MA, Khoury MJ, Macleod MR, Moher D, Schulz KF and Tibshirani R: Increasing value and reducing waste in research design, conduct, and analysis. Lancet 383(9912): 166-175, 2014.

56 International Committee of Medical Journal Editors, Drazen JM, Van Der Weyden MB, Sahni P, Rosenberg J, Marusic A, Laine C, Kotzin S, Horton R, Hébert PC, Haug C, Godlee F, Frizelle FA, de Leeuw PW and DeAngelis CD: Uniform format for disclosure of competing interests in ICMJE journals. N Engl J Med 361(19): 1896-1897, 2009.

57 Schulz KF, Altman DG, Moher D and CONSORT Group: CONSORT 2010 statement: updated guidelines for reporting parallel group randomised trials. BMJ (Clinical research ed) 340: c332, 2010.

58 Editors PM: From checklists to tools: lowering the barrier to better research reporting. PLoS Med 12(11): e1001910, 2015.

59 Barnes C, Boutron I, Giraudeau B, Porcher R, Altman DG and Ravaud P: Impact of an online writing aid tool for writing a randomized trial report: the COBWEB (Consort-based WEB tool) randomized controlled trial. BMC Med 13: 221, 2015.

Received June 2, 2017

Revised July 15, 2017

Accepted July 19, 2017 